\title{
MULTIPLIER CRITERIA OF MARCINKIEWICZ TYPE FOR JACOBI EXPANSIONS
}

\author{
BY
}

GEORGE GASPER(1) AND WALTER TREBELS

\begin{abstract}
It is shown how an integral representation for the product of Jacobi polynomials can be used to derive a certain integral Lipschitz type condition for the Cesàro kernel for Jacobi expansions. This result is then used to give criteria of Marcinkiewicz type for a sequence to be multiplier of type $(p, p), 1<p<\infty$, for Jacobi expansions.
\end{abstract}

1. Introduction. Fix $\alpha \geqslant \beta \geqslant-\frac{1}{2}$ and let $L^{p}=L_{(\alpha, \beta)}^{p}, 1 \leqslant p<\infty$, denote the space of measurable functions $f(\theta)$ on $[0, \pi]$ for which

$$
\|f\|_{p}=\left(\int_{0}^{\pi}|f(\theta)|^{p} d \mu(\theta)\right)^{1 / p}<\infty
$$

where

$$
d \mu(\theta)=d \mu^{(\alpha, \beta)}(\theta)=\left(\sin \frac{\theta}{2}\right)^{2 \alpha+1}\left(\cos \frac{\theta}{2}\right)^{2 \beta+1} d \theta .
$$

Also let

$$
R_{k}(x)=R_{k}^{(\alpha, \beta)}(x)=P_{k}^{(\alpha, \beta)}(x) / P_{k}^{(\alpha, \beta)}(1),
$$

where $P_{k}^{(\alpha, \beta)}(x)$ is the Jacobi polynomial of order $(\alpha, \beta)[16]$. Each $f \in L^{p}$ has the Jacobi expansion

$$
f(\theta) \sim \sum_{k=0}^{\infty} f^{\wedge}(k) h_{k} R_{k}(\cos \theta)
$$

where

Presented to the Society, August 25, 1976; received by the editors January 5, 1976.

AMS (MOS) subject classifications (1970). Primary 42A18, 42A56; Secondary 33A45, 33A65, 40G05.

Key words and phrases. Multipliers, Jacobi polynomials, ultraspherical polynomials, Cesàro kernel, Poisson kernel, fractional differences.

(1) Supported in part by NSF Grant MPS 71-03407 A03 and in part by a fellowship from the Alfred P. Sloan foundation. 


$$
\begin{aligned}
f^{\wedge}(k) & =\int_{0}^{\pi} f(\theta) R_{k}(\cos \theta) d \mu(\theta), \\
h_{k} & =h_{k}^{(\alpha, \beta)}=\left\{\int_{0}^{\pi}\left(R_{k}(\cos \theta)\right)^{2} d \mu(\theta)\right\}^{-1} \\
& =\frac{(2 k+\alpha+\beta+1) \Gamma(k+\alpha+\beta+1) \Gamma(k+\alpha+1)}{\Gamma(k+\beta+1) \Gamma(k+1) \Gamma(\alpha+1) \Gamma(\alpha+1)} .
\end{aligned}
$$

A sequence $\eta=\left\{\eta_{k}\right\}_{k=0}^{\infty}$ is called a multiplier of type $(p, q)$, notation $\eta \in M_{p}^{q}$, if for each $f \in L^{p}$ there exists a function $f^{\eta} \in L^{q}$ with

$$
f^{\eta}(\theta) \sim \sum_{k=0}^{\infty} \eta_{k} f^{\wedge}(k) h_{k} R_{k}(\cos \theta)
$$

and $\left\|f^{\eta}\right\|_{q} \leqslant C_{p, q, \eta}\|f\|_{p}$. Here, as elsewhere, $C$ is a positive constant, not always the same at different occasions, which depends only on the parameters indicated by subscripts, except that the dependence on the parameters $\alpha, \beta$, or $\gamma$ will not be indicated. Also, we will let $\int \cdots d \mu(\theta)$ denote integration over the interval $[0, \pi]$.

Our aim is to give sufficient multiplier criteria of Marcinkiewicz type for a sequence to belong to $M_{p}^{p}, 1<p<\infty$. For this purpose we consider the fractional difference operator $\Delta^{\lambda}$ defined by [4]

$$
\Delta^{\lambda} \eta_{k}=\sum_{j=k}^{\infty} A_{j-k}^{-\lambda-1} \eta_{j}, \quad A_{k}^{\lambda}=\left(\begin{array}{c}
k+\lambda \\
k
\end{array}\right)=\frac{\Gamma(k+\lambda+1)}{\Gamma(k+1) \Gamma(\lambda+1)},
$$

whenever the series converges, and for $\gamma \geqslant 0$ we define the space wbv $_{\gamma+1}$ of sequences of weak bounded variation of order $\gamma+1$ by

$$
\operatorname{wbv}_{\gamma+1}=\left\{\eta \in l^{\infty}:\|\eta\|_{\gamma+1, w}=\|\eta\|_{\infty}+\sup _{m \geqslant 1} \sum_{2^{m-1}}^{2^{m}-1} A_{k}^{\gamma}\left|\Delta^{\gamma+1} \eta_{k}\right|<\infty\right\},
$$

where $l^{\infty}$ denotes the space of bounded sequences $\eta=\left\{\eta_{k}\right\}_{k=0}^{\infty}$ with the sup norm $\|\eta\|_{\infty}$. The space $b_{\gamma+1}$ of sequences of bounded variation of order $\gamma+1$ is defined as above except that $k$ runs from 0 to $\infty$, see [17, p. 20]. Our main result can then be stated as follows.

THEOREM 1. If $\alpha \geqslant \beta \geqslant-\frac{1}{2}, \gamma>\alpha+\frac{1}{2}$, and $\eta \in \mathrm{wbv}_{\gamma+1}$, i.e.

$$
\|\eta\|_{\infty}+\sup _{m} \sum_{2^{m-1}}^{2^{m}-1} A_{k}^{\gamma}\left|\Delta^{\gamma+1} \eta_{k}\right|<\infty,
$$

then $\eta \in M_{p}^{p}, 1<p<\infty$.

Recently, Connnett and Schwartz [6], [7] proved essentially 
THEOREM A. Let $\alpha \geqslant \beta>-\frac{1}{2}$ and $\gamma=[\alpha+1]$, where $[\alpha+1]$ is the integer part of $\alpha+1$. If

$$
\|\eta\|_{\infty}+\sup _{m}\left(\sum_{2^{m-1}}^{2^{m-1}} k^{-1}\left|A_{k}^{\gamma+1} \Delta^{\gamma+1} \eta_{k}\right|^{2}\right)^{1 / 2}<\infty,
$$

then $\eta \in M_{p}^{p}, 1<p<\infty$.

By Schwarz's inequality it follows that Theorem 1 implies Theorem A when $[\alpha+1]>\alpha+\frac{1}{2}$. Let us mention that if $\gamma>\alpha+\frac{1}{2}$ then

$$
\|\eta\|_{\infty}+\sum_{k=0}^{\infty} A_{k}^{\gamma}\left|\Delta^{\gamma+1} \eta_{k}\right|<\infty
$$

is a sufficient condition for $\eta \in M_{1}^{1}$, see [17, pp. 21, 88]. For $M_{p}^{q}$-multipliers, $1 \leqslant p<q \leqslant \infty$, see [2]. Also see Butzer, Nessel, Trebels [17, ref. 33].

For the proof of Theorem 1 we need two useful lemmas concerning the space wbv $_{\gamma+1}$, which will be proved in $\$ \S 2$ and 3 .

LEMMA 1. If $0 \leqslant \gamma^{\prime}<\gamma$ then wbv $_{\gamma+1} \subset \mathrm{wbv}_{\gamma^{\prime}+1}$, where the inclusion sign means that the identity map is continuous.

Lemma 2. Let $G(t) \in C^{\infty}[0, \infty)$ be monotone decreasing with $G(t)=1$ if $t \leqslant 1$ and $G(t)=0$ if $t \geqslant 2$. If $\gamma \geqslant 0$ and $u>0$, then

$$
\left\|\left\{\eta_{k} G(k / u)\right\}\right\|_{\gamma+1, w} \leqslant C\|\eta\|_{\gamma+1, w}
$$

with $C$ independent of $u$.

In particular, it should be noted that, since

$$
R_{k}^{(-1 / 2,-1 / 2)}(\cos \theta)=\cos k \theta,
$$

the case $\alpha=\beta=-\frac{1}{2}$ of Theorem 1 follows directly from Lemma 1 and the classical Marcinkiewicz multiplier theorem [14], [18, p. 232]. In \$4 we shall use these lemmas and the observations in Coifman and Weiss $[5, \mathrm{pp} .74,75]$ to reduce the proof of Theorem 1 to showing that a certain kernel satisfies a uniform Hörmander condition and to show that this condition follows from the following proposition, which is the crucial part of this paper.

Proposition 1. Let $\alpha \geqslant \beta \geqslant-\frac{1}{2}$ and $\alpha+\frac{1}{2}<\gamma \leqslant \alpha+\frac{3}{2}$. Then the Cesàro kernel

$$
\sigma_{n}^{\gamma}(\theta, \varphi)=\sum_{k=0}^{n} \frac{A_{n-k}^{\gamma}}{A_{n}^{\gamma}} h_{k} R_{k}(\cos \theta) R_{k}(\cos \varphi)
$$

satisfies the inequalities 


$$
\begin{gathered}
\int\left|\sigma_{n}^{\gamma}(\theta, \varphi)-\sigma_{n}^{\gamma}\left(\theta, \varphi_{0}\right)\right| d \mu(\theta) \leqslant C n\left|\varphi-\varphi_{0}\right| \\
\int\left|\sigma_{n}^{\gamma}(\theta, \varphi)\right|(n|\theta-\varphi|)^{\varepsilon} d \mu(\theta) \leqslant C_{\varepsilon}, \quad 0<\varepsilon<\gamma-\alpha-\frac{1}{2} .
\end{gathered}
$$

Our proof of (1.3) in $\S 5$ is of special interest since it is quite different from and much simpler computationally than that which Connett and Schwartz used in [6], [7] to prove (with a slightly different notation) that if $\alpha \geqslant \beta>-\frac{1}{2}$, then the Poisson kernel

$$
W_{r}(\theta, \varphi)=\sum_{k=0}^{\infty}(1-r)^{k} h_{k} R_{k}(\cos \theta) R_{k}(\cos \varphi)
$$

satisfies the inequality

$$
\int\left|W_{r}(\theta, \varphi)-W_{r}\left(\theta, \varphi_{0}\right)\right| d \mu(\theta) \leqslant \frac{C}{r}\left|\varphi-\varphi_{0}\right| .
$$

Recall that for the ultraspherical case $\alpha=\beta>-\frac{1}{2}$ in [6] they used the Muckenhoupt and Stein [15] bounds for the kernel (1.5) and an integral representation of Watson to derive bounds for a partial derivative of (1.5), and then used these bounds, the Mean Value Theorem, and Watson's integral to prove (1.6); while for the case $\alpha>\beta>-\frac{1}{2}$ the main computations in [7] were directed at the derivation of bounds for (1.5) and a partial derivative of it. Our method for proving (1.3) uses both of the integral representations for the product of Jacobi polynomials in Koornwinder [13] and Gasper [9], [10] in such a way that, instead of having to derive and use bounds for a partial derivative of a two-variable kernel, we only need to consider the derivative of a one-variable kernel. In $\S 6$ we shall show that this method also yields a very short proof of (1.6) for $\alpha \geqslant \beta \geqslant-\frac{1}{2}$, and hence an extension of Theorem $A$ to $\alpha \geqslant \beta \geqslant-\frac{1}{2}$.

2. Proof of Lemma 1. First note that for $\eta \in l^{\infty}$ we have

$$
\Delta^{a}\left(\Delta^{b} \eta_{k}\right)=\Delta^{a+b} \eta_{k}
$$

if $a>-1, b \geqslant 0, a+b>0$; see [4, Lemma 1]. If $\gamma^{\prime} \geqslant 0, \gamma-1<\gamma^{\prime}<\gamma$, and we set $d=\gamma-\gamma^{\prime}$, then $0<d<1$ and, by (2.1),

$$
\begin{aligned}
\sum_{k=2^{m-1}}^{2^{m}-1} A_{k}^{\gamma^{\prime}}\left|\Delta^{\gamma^{\prime}+1} \eta_{k}\right| & \leqslant \sum_{2^{m-1}}^{2^{m}-1} A_{k}^{\gamma-d} \sum_{j=k}^{\infty} A_{j-k}^{d-1}\left|\Delta^{\gamma+1} \eta_{j}\right| \\
& =\sum_{k=2^{m-1}}^{2^{m}-1}\left(\sum_{j=k}^{2^{m}-1}+\sum_{j=2^{m}}^{\infty}\right)=\mathrm{I}_{1}+\mathrm{I}_{2} .
\end{aligned}
$$

A change in the order of summation in $I_{1}$ gives 


$$
\mathrm{I}_{1}=\sum_{j=2^{m-1}}^{2^{m}-1}\left|\Delta^{\gamma+1} \eta_{j}\right| \sum_{k=2^{m-1}}^{j} A_{k}^{\gamma-d} A_{j-k}^{d-1} \leqslant\|\eta\|_{\gamma+1, w}
$$

uniformly in $m$. Also

$$
\begin{aligned}
\mathrm{I}_{2} & =\sum_{2^{m-1}}^{2^{m}-1} A_{k}^{\gamma-d} \sum_{i=m+1}^{\infty} \sum_{j=2^{i-1}}^{2^{i}-1} A_{j-k}^{d-1}\left|\Delta^{\gamma+1} \eta_{j}\right| \\
& \leqslant C\|\eta\|_{\gamma+1, w} \sum_{i=m+1}^{\infty}\left(2^{i-1}\right)^{-\gamma} \sum_{k=2^{m-1}}^{2^{m}-1} A_{k}^{\gamma-d} A_{2^{m}-1-k}^{d-1} \\
& \leqslant C\|\eta\|_{\gamma+1, w}\left(2^{m}\right)^{\gamma} \sum_{i=m}^{\infty}\left(2^{i}\right)^{-\gamma} \leqslant C\|\eta\|_{\gamma+1, w}
\end{aligned}
$$

uniformly in $m$. Hence Lemma 1 follows if $\gamma^{\prime} \geqslant 0$ is such that $\gamma-1<\gamma^{\prime}$ $\langle\gamma$. If $\gamma-1 \geqslant 0$ then one need but iterate the previous case finitely often, and thus Lemma 1 is proved.

3. Proof of Lemma 2. We first consider integer $\gamma$. Then Leibniz' formula gives

$$
\Delta^{\gamma+1} \eta_{k} G\left(\frac{k}{u}\right)=\sum_{j=0}^{\gamma+1}\left(\begin{array}{c}
\gamma+1 \\
j
\end{array}\right)\left(\Delta^{j} \eta_{k}\right) \Delta^{\gamma+1-j} G\left(\frac{j+k}{u}\right) .
$$

By $[17$, p. 26] and the hypotheses on $G(u)$,

$$
\sum_{k=0}^{\infty} A_{k}^{j}\left|\Delta^{j+1} G\left(\frac{k}{u}\right)\right| \leqslant \int_{0}^{\infty} t^{j}\left|G^{(j+1)}(t)\right| d t \leqslant C_{j}
$$

for $j=0,1, \ldots$ Hence

$$
\left|k^{j} \Delta^{j} G\left(\frac{k}{u}\right)\right|=\left|k^{j} \sum_{i=k}^{\infty} \Delta^{j+1} G\left(\frac{i}{u}\right)\right| \leqslant C_{j} \sum_{i=0}^{\infty} A_{i}^{j}\left|\Delta^{j+1} G\left(\frac{i}{u}\right)\right| \leqslant C_{j}
$$

and so, using the above and Lemma 1, we have

$$
\begin{aligned}
\sum_{k=2^{m-1}}^{2^{m}-1} A_{k}^{\gamma}\left|\Delta^{\gamma+1} \eta_{k} G\left(\frac{k}{u}\right)\right| \leqslant & \sum_{2^{m-1}}^{2^{m}-1}\left|\eta_{k}\right| A_{k}^{\gamma}\left|\Delta^{\gamma+1} G\left(\frac{k}{u}\right)\right| \\
& +\sum_{j=1}^{\gamma+1}\left(\begin{array}{c}
\gamma+1 \\
j
\end{array}\right) \frac{\Gamma(j)}{\Gamma(\gamma+1)} \sum_{k=2^{m-1}}^{2^{m-1}} A_{k}^{j-1}\left|\Delta^{j} \eta_{k}\right| \\
& \cdot\left\{(\gamma+k) \cdots(j+k)\left|\Delta^{\gamma+1-j} G\left(\frac{j+k}{u}\right)\right|\right\} \\
\leqslant & C\left\{\|\eta\|_{\infty}+\sum_{j=1}^{\gamma+1}\|\eta\|_{j, w}\right\} \leqslant C\|\eta\|_{\gamma+1, w}
\end{aligned}
$$


uniformly in $m$ and $u>0$. Therefore the lemma is proved for integer $\gamma$.

For positive noninteger values of $\gamma$ we set $a=[\gamma]$. Then, by (2.1) and (3.1),

$$
\begin{aligned}
\Delta^{\gamma+1}\left(\eta_{k} G\left(\frac{k}{u}\right)\right)= & \Delta \Delta^{\gamma-a-1} \sum_{j=0}^{a+1}\left(\begin{array}{c}
a+1 \\
j
\end{array}\right)\left(\Delta^{j} \eta_{k}\right) \Delta^{a+1-j} G\left(\frac{j+k}{u}\right) \\
= & \Delta \sum_{i=k}^{a+1} A_{i-k}^{a-\gamma} G\left(\frac{i+a+1}{u}\right) \Delta^{a+1} \eta_{i} \\
& +\Delta \sum_{j=0}^{a}\left(\begin{array}{c}
a+1 \\
j
\end{array}\right) \sum_{i=k}^{\infty} A_{i-k}^{a-\gamma}\left(\Delta^{j} \eta_{i}\right) \Delta^{a+1-j} G\left(\frac{i+j}{u}\right) \\
= & \Sigma_{1}+\Sigma_{2} .
\end{aligned}
$$

By (2.1),

$$
\Delta^{a+1} \eta_{i}=\sum_{j=i}^{\infty} A_{j-i}^{\gamma-a-1} \Delta^{\gamma+1} \eta_{j}
$$

and hence, after a change in order of summation,

$$
\begin{aligned}
\Sigma_{1} & =\Delta \sum_{j=k}^{\infty} \Delta^{\gamma+1} \eta_{j} \sum_{i=k}^{j} A_{i-k}^{a-\gamma} A_{j-i}^{\gamma-a-1} G\left(\frac{i+a+1}{u}\right) \\
& =\lambda_{k}^{(k)} \Delta^{\gamma+1} \eta_{k}+\sum_{j=k+1}^{\infty}\left(\lambda_{k}^{(j)}-\lambda_{k+1}^{(j)}\right) \Delta^{\gamma+1} \eta_{j},
\end{aligned}
$$

where

$$
\lambda_{k}^{(j)}= \begin{cases}\sum_{i=k}^{j} A_{i-k}^{a-\gamma} A_{j-i}^{\gamma-a-1} G\left(\frac{i+a+1}{u}\right), & k \leqslant j \\ 0, & k>j .\end{cases}
$$

It is not hard to check that $\left\{\lambda_{k}^{(j)}\right\}_{k=0}^{\infty}$ is monotone decreasing and bounded by 1 . In particular

$$
\sum_{k=0}^{\infty}\left|\Delta \lambda_{k}^{(j)}\right| \leqslant 1
$$

uniformly in $j$ and $u>0$. Therefore, 


$$
\begin{aligned}
\sum_{2^{m-1}}^{2^{m}-1} A_{k}^{\gamma}\left|\Sigma_{1}\right| \leqslant & \sum_{2^{m-1}}^{2^{m}-1} A_{k}^{\gamma}\left|\Delta^{\gamma+1} \eta_{k}\right|+\sum_{2^{m-1}}^{2^{m}-1} A_{k}^{\gamma} \sum_{j=k+1}^{2^{m}-1}\left|\Delta \lambda_{k}^{(j)}\right|\left|\Delta^{\gamma+1} \eta_{j}\right| \\
& +\sum_{2^{m-1}}^{2^{m}-1} A_{k}^{\gamma} \sum_{i=m+1}^{\infty} \sum_{j=2^{i-1}}^{2^{i}-1}\left|\Delta \lambda_{k}^{(j)}\right|\left|\Delta^{\gamma+1} \eta_{j}\right| \\
\leqslant & \|\eta\|_{\gamma+1, w}+C \sum_{j=2^{m-1}}^{2^{m}-1} A_{j}^{\gamma}\left|\Delta^{\gamma+1} \eta_{j}\right| \sum_{k=2^{m-1}}^{j-1}\left|\Delta \lambda_{k}^{(j)}\right| \\
& +C \sum_{i=m+1}^{\infty}\left(2^{i-1}\right)^{-\gamma} \sum_{j=2^{i-1}}^{2^{i}-1} A_{j}^{\gamma}\left|\Delta^{\gamma+1} \eta_{j}\right| \sum_{k=2^{m-1}}^{2^{m}-1} A_{k}^{\gamma}\left|\Delta \lambda_{k}^{(j)}\right| \\
\leqslant & C\|\eta\|_{\gamma+1, w}\left\{1+\left(2^{m}\right)^{\gamma} \sum_{i=m}^{\infty}\left(2^{i}\right)^{-\gamma}\right\} \leqslant C\|\eta\|_{\gamma+1, w} .
\end{aligned}
$$

For $\Sigma_{2}$ we observe that

$$
\begin{aligned}
\sum^{2^{m-1}} A_{k}^{\gamma} \mid & \Sigma_{2} \mid \\
= & \sum_{2^{m-1}}^{2^{m}-1} A_{k}^{\gamma} \mid \sum_{j=0}^{a}\left(\begin{array}{c}
a+1 \\
j
\end{array}\right) \sum_{i=k}^{\infty} A_{i-k}^{a-\gamma}\left\{\left(\Delta^{j} \eta_{i}\right) \Delta^{a+2-j} G\left(\frac{i+j}{u}\right)\right. \\
& \left.+\left(\Delta^{j+1} \eta_{i}\right) \Delta^{a+1-j} G\left(\frac{i+j+1}{u}\right)\right\} \mid \\
= & \sum_{2^{m-1}}^{2^{m}-1} A_{k}^{\gamma}\left|\sum_{j=0}^{a+1} C_{j, a} \sum_{i=k}^{\infty} A_{i-k}^{a-\gamma}\left(\Delta^{j} \eta_{i}\right) \Delta^{a+2-j} G\left(\frac{i+j}{u}\right)\right| \\
\leqslant & C \sum_{k=2^{m-1}}^{2^{m}-1} A_{k}^{\gamma} \sum_{i=k}^{\infty} A_{i-k}^{a-\gamma}\left|\eta_{i} \Delta^{a+2} G\left(\frac{i}{u}\right)\right| \\
& +C \sum_{j=1}^{a+1} \sum_{k=2^{m-1}}^{2^{m}-1} A_{k}^{\gamma}\left(\sum_{i=k}^{2^{m}-1}+\sum_{s=m+1}^{\infty} \sum_{i=2^{s-1}}^{2^{s}-1}\right) \\
\leqslant & C\|\eta\|_{\infty} \sum_{i=2^{m-1}}^{\infty}\left|\Delta^{a+2} G\left(\frac{i}{u}\right)\right| \sum_{k=2^{m-1}}^{i} A_{k}^{\gamma} A_{i-k}^{a-\gamma} \\
& +C \sum_{j=1}^{a+1} \sum_{i=2^{m-1}}^{a-\gamma}\left|\left(\Delta^{j} \eta_{i}\right) \Delta^{a+2-j} G\left(\frac{i+j}{u}\right)\right| \\
& \cdot \sum_{k=2^{m-1}}^{i} A_{i-k}^{a-\gamma} A_{k}^{\gamma+j-a-2}\left\{k^{a+2-j}\left|\Delta^{a+2-j} G\left(\frac{i+j}{u}\right)\right|\right\}
\end{aligned}
$$




$$
\begin{aligned}
& +C \sum_{j=1}^{a+1} \sum_{s=m+1}^{\infty} \sum_{i=2^{s-1}}^{2^{s-1}}\left|\Delta^{j} \eta_{i}\right| \cdot\left|\Delta^{a+2-j} G\left(\frac{i+j}{u}\right)\right| \sum_{k=2^{m-1}}^{2^{m}-1} A_{k}^{\gamma} A_{2^{m}-1-k}^{a-\gamma} \\
& \leqslant C\|\eta\|_{\infty} \sum_{i=0}^{\infty} A_{i}^{a+1}\left|\Delta^{a+2} G\left(\frac{i}{u}\right)\right|+C \sum_{j=1}^{a+1} \sum_{i=2^{m-1}}^{2^{m}-1} A_{i}^{j-1}\left|\Delta^{j} \eta_{i}\right| \\
& +C \sum_{j=1}^{a+1}\left(2^{m}\right)^{j} \sum_{s=m+1}^{\infty}\left(2^{s-1}\right)^{-j} \\
& \quad \cdot \sum_{i=2^{s-1}}^{2^{s-1}} A_{i}^{j-1}\left|\Delta^{j} \eta_{i}\right|\left\{i^{a+2-j}\left|\Delta^{a+2-j} G\left(\frac{i+j}{u}\right)\right|\right\} \\
& \leqslant C\|\eta\|_{\gamma+1, w},
\end{aligned}
$$

by our previous observations, which completes the proof.

4. Proof of Theorem 1. In view of the Marcinkiewicz multiplier theorem and Lemma 1 we may assume that $\alpha \geqslant \beta \geqslant-\frac{1}{2}, \alpha>-\frac{1}{2}$, and $\alpha+\frac{1}{2}<\gamma<\alpha$ $+\frac{3}{2}$. Since the sums

$$
S_{n}(\theta)=\sum_{k=0}^{n} c_{k} R_{k}(\cos \theta)
$$

are dense in $L^{p}, 1<p<\infty$, it suffices to show that

$$
\left\|S_{n}^{\eta}\right\|_{p} \leqslant C_{p}\left\|S_{n}\right\|_{p}, \quad 1<p<\infty
$$

with $C_{p}$ independent of $S_{n}$. To prove this we will need the following convolution structure for Jacobi series.

It was proved in [9], [10] that if $\alpha \geqslant \beta \geqslant-\frac{1}{2}$ and $\alpha>-\frac{1}{2}$, then there is a nonnegative function $K(\theta, \varphi, \psi)$, symmetric in $\theta, \varphi, \psi$, such that

$$
R_{n}(\cos \theta) R_{n}(\cos \varphi)=\int R_{n}(\cos \psi) K(\theta, \varphi, \psi) d \mu(\psi)
$$

and

$$
\int K(\theta, \varphi, \psi) d \mu(\psi)=1
$$

for $0<\theta, \varphi<\pi$. From this it follows that the generalized translation operator $T_{\varphi}$ defined by

$$
\begin{aligned}
T_{\varphi} f(\theta) & =\int f(\psi) K(\theta, \varphi, \psi) d \mu(\psi) \\
& \sim \sum_{k=0}^{\infty} f^{\wedge}(k) h_{k} R_{k}(\cos \theta) R_{k}(\cos \varphi)
\end{aligned}
$$


is a bounded operator on $L^{1}$, and that with the convolution of two functions in $L^{1}$ defined by

$$
\begin{aligned}
(f * g)(\theta) & =\int f(\varphi)\left(T_{\varphi} g(\theta)\right) d \mu(\varphi) \\
& =\iint f(\varphi) g(\psi) K(\theta, \varphi, \psi) d \mu(\psi) d \mu(\varphi)
\end{aligned}
$$

we have $(f * g)^{\wedge}(k)=f^{\wedge}(k) g^{\wedge}(k)$.

Hence, with $G(t)$ as in Lemma 2 ,

$$
S_{n}^{\eta}(\theta)=\left(S_{n} * \chi_{u}\right)(\theta), \quad u \geqslant n,
$$

where

$$
\chi_{u}(\theta)=\sum_{k=0}^{\infty} \eta_{k} G\left(\frac{k}{u}\right) h_{k} R_{k}(\cos \theta), \quad u>0,
$$

and so in proving (4.1) we may restrict ourselves to a (uniform in $u$ ) discussion of the kernel (4.4). On account of the observations in Coifman and Weiss [5, pp. 74, 75] it suffices to show that the generalized translation $T_{\varphi} \chi_{u}(\theta)$ satisfies the following version of Hörmander's condition [12] (see also Connett and Schwartz [6], [7]):

$$
\int_{E}\left|T_{\varphi} \chi_{u}(\theta)-T_{\varphi_{0}} \chi_{u}(\theta)\right| d \mu(\theta) \leqslant C
$$

where $E=\left\{\theta:\left|\theta-\varphi_{0}\right|>2\left|\varphi-\varphi_{0}\right|\right\}$ and $C$ is independent of $u$.

By a change in order of summation (cf. [17, p. 22]), for each $u>0$ we can represent $T_{\varphi} \chi_{u}(\theta)$ by

$$
T_{\varphi} \chi_{u}(\theta)=\sum_{n=0}^{\infty} A_{n}^{\gamma}\left(\Delta^{\gamma+1}\left(\eta_{n} G\left(\frac{n}{u}\right)\right)\right) \sigma_{n}^{\gamma}(\theta, \varphi),
$$

where $\sigma_{n}^{\gamma}(\theta, \varphi)$ is the Cesàro kernel (1.2). Hence,

$$
\begin{aligned}
& \int_{E}\left|T_{\varphi} \chi_{u}(\theta)-T_{\varphi_{0}} \chi_{u}(\theta)\right| d \mu(\theta) \\
& \leqslant \sum_{m=1}^{\infty} \sum_{n=2^{m-1}}^{2^{m}-1} A_{n}^{\gamma}\left|\Delta^{\gamma+1}\left(\eta_{n} G\left(\frac{n}{u}\right)\right)\right| \int_{E}\left|\sigma_{n}^{\gamma}(\theta, \varphi)-\sigma_{n}^{\gamma}\left(\theta, \varphi_{0}\right)\right| d \mu(\theta) \\
& \leqslant\left\|\left\{\eta_{k} G\left(\frac{k}{u}\right)\right\}\right\| \|_{\gamma+1, w} \sum_{m=1}^{\infty} \sup _{2^{m-1} \leqslant n<2^{m}} \int_{E}\left|\sigma_{n}^{\gamma}(\theta, \varphi)-\sigma_{n}^{\gamma}\left(\theta, \varphi_{0}\right)\right| d \mu(\theta) \\
& \leqslant C\|\eta\|_{\gamma+1, w}\left(\sum_{m=0}^{m_{0}}+\sum_{m=m_{0}+1}^{\infty}\right)_{2^{m} \leqslant n<2^{m+1}} \sup _{E}\left|\sigma_{n}^{\gamma}(\theta, \varphi)-\sigma_{n}^{\gamma}\left(\theta, \varphi_{0}\right)\right| d \mu(\theta),
\end{aligned}
$$

where we have chosen $m_{0}$ such that 


$$
2^{m_{0}-1}\left|\varphi-\varphi_{0}\right| / \pi<1 \leqslant 2^{m_{0}}\left|\varphi-\varphi_{0}\right| / \pi
$$

Then

$$
2^{m-m_{0}} \leqslant n\left|\varphi-\varphi_{0}\right| / \pi<2^{m-m_{0}+2} \text { when } 2^{m} \leqslant n<2^{m+1},
$$

and so to complete the proof one need but observe that by Proposition 1 (proved below) both sums in (4.6) are bounded independent of the choice of $\varphi$ and $\varphi_{0}$.

Corresponding to Connett and Schwartz [7], it also follows from (4.5), under the conditions of Theorem 1 , that $\eta$ is of weak type $(1,1)$.

5. Proof of Proposition 1. Let us first consider the easier inequality (1.4) for $\alpha \geqslant \beta \geqslant-\frac{1}{2}, \alpha>-\frac{1}{2}$. Then, by (4.2),

$$
\sigma_{n}^{\gamma}(\theta, \varphi)=\int \sigma_{n}^{\gamma}(\psi) K(\theta, \varphi, \psi) d \mu(\psi)
$$

where $\sigma_{n}^{\gamma}(\psi)=\sigma_{n}^{\gamma}(\psi, 0)$. Using a standard method and the fact that $K(\theta, \varphi, \psi)$ $=0$ unless $|\theta-\varphi|<\psi$ (see [10, p. 268]) we have, for $0<\varepsilon<\gamma-\alpha-\frac{1}{2}$ $\leqslant 1$,

$$
\begin{aligned}
\int\left|\sigma_{n}^{\gamma}(\theta, \varphi)\right| & (n|\theta-\varphi|)^{\varepsilon} d \mu(\theta) \\
& \leqslant \iint\left|\sigma_{n}^{\gamma}(\psi)\right| K(\theta, \varphi, \psi)(n|\theta-\varphi|)^{\varepsilon} d \mu(\psi) d \mu(\theta) \\
& \leqslant \int\left|\sigma_{n}^{\gamma}(\psi)\right|(n \psi)^{\varepsilon}\left\{\int K(\theta, \varphi, \psi) d \mu(\theta)\right\} d \mu(\psi) \\
& =\int\left|\sigma_{n}^{\gamma}(\psi)\right|(n \psi)^{\varepsilon} d \mu(\psi) \leqslant C_{\varepsilon}
\end{aligned}
$$

by means of (4.3) and the Bonami and Clerc [3, p. 230] estimates

$$
\mid \sigma_{n}^{\gamma}(\psi) \leqslant \begin{cases}C n^{2 \alpha+2}, & 0 \leqslant \psi \leqslant \pi / 2, \\ C n^{\alpha+1 / 2-\gamma} \psi^{-\alpha-\gamma-3 / 2}, & 2 / n \leqslant \psi \leqslant \pi / 2, \\ C n^{\alpha+1 / 2-\gamma}(\pi-\psi)^{-\beta-1 / 2}, & \pi / 2 \leqslant \psi \leqslant \pi-2 / n, \\ C n^{\alpha+\beta+1-\gamma}, & \pi / 2 \leqslant \psi \leqslant \pi,\end{cases}
$$

which hold for $\gamma \leqslant \alpha+\frac{3}{2}, \alpha \geqslant \beta \geqslant-\frac{1}{2}$. Similarly, the case $\alpha=\beta=-\frac{1}{2}$ of (1.4) follows by using the identity

$$
\begin{aligned}
R_{n}^{(-1 / 2,-1 / 2)} & (\cos \theta) R_{n}^{(-1 / 2,-1 / 2)}(\cos \varphi) \\
& =\frac{1}{2} R_{n}^{(-1 / 2,-1 / 2)}(\cos (\theta+\varphi))+\frac{1}{2} R_{n}^{(-1 / 2,-1 / 2)}(\cos (\theta-\varphi))
\end{aligned}
$$

in place of the product formula (4.2). 
We shall now prove (1.3). In [13] Koornwinder showed that if $\alpha>\beta>-\frac{1}{2}$, then for the right side of (4.2) we have that

$$
\int_{0}^{\pi} R_{n}(\cos \psi) K(\theta, \varphi, \psi) d \mu(\psi)=\int_{0}^{\pi} \int_{0}^{1} R_{n}(\cos \Psi) d m(r, t),
$$

where

(5.5) $d m(r, t)=\frac{2 \Gamma(\alpha+1)}{\Gamma\left(\frac{1}{2}\right) \Gamma(\alpha-\beta) \Gamma\left(\beta+\frac{1}{2}\right)}\left(1-r^{2}\right)^{\alpha-\beta-1} r^{2 \beta+1}(\sin t)^{2 \beta} d r d t$,

$$
\begin{aligned}
\cos \Psi= & \cos \theta \cos \varphi+r \sin \theta \sin \varphi \cos t \\
& +\frac{1}{2}\left(r^{2}-1\right)(1-\cos \theta)(1-\cos \varphi),
\end{aligned}
$$

and he also showed that (5.4) holds with $R_{n}$ replaced by an arbitrary continuous function.

Our proof of (1.3) depends at a crucial step on the observation that with $\cos \Psi$ defined by (5.6) and $0 \leqslant r \leqslant 1,0 \leqslant t, \theta, \varphi \leqslant \pi$, we have the inequality

$$
\left|\frac{\partial \cos \Psi}{\partial \varphi}\right| \leqslant C(1-\cos \Psi)^{1 / 2} \text {. }
$$

Clearly,

$$
\begin{aligned}
1-\cos \Psi= & 1-\cos (\theta-\varphi)+(1-r \cos t) \sin \theta \sin \varphi \\
& +\frac{1}{2}\left(1-r^{2}\right)(1-\cos \theta)(1-\cos \varphi),
\end{aligned}
$$

and

$\frac{\partial \cos \Psi}{\partial \varphi}=\sin (\theta-\varphi)+(r \cos t-1) \sin \theta \cos \varphi+\frac{1}{2}\left(r^{2}-1\right)(1-\cos \theta) \sin \varphi$.

Hence, by (5.7),

$$
\begin{aligned}
&|\sin (\theta-\varphi)|=(1+\cos (\theta-\varphi))^{1 / 2}(1-\cos (\theta-\varphi))^{1 / 2} \\
& \leqslant 2^{1 / 2}(1-\cos \Psi)^{1 / 2} \\
&\left|\frac{1}{2}\left(r^{2}-1\right)(1-\cos \theta) \sin \varphi\right| \leqslant C\left\{\left(1-r^{2}\right)(1-\cos \theta)(1-\cos \varphi)\right\}^{1 / 2} \\
& \leqslant C(1-\cos \Psi)^{1 / 2} .
\end{aligned}
$$

If $\varphi \leqslant \theta / 2$ then $\sin |(\theta-\varphi) / 2| \geqslant \sin \theta / 4$, while if $\varphi \geqslant(\pi+\theta) / 2$ then $\sin |(\theta-\varphi) / 2| \geqslant \sin (\pi-\theta) / 4$, and so in either case 
$|(r \cos t-1) \sin \theta \cos \varphi| \leqslant 2 \sin \theta\left(\frac{1-\cos \Psi}{1-\cos (\theta-\varphi)}\right)^{1 / 2}$

$$
=C(1-\cos \Psi)^{1 / 2} \frac{\sin \theta}{\sin |(\theta-\varphi) / 2|} \leqslant C(1-\cos \Psi)^{1 / 2} \text {. }
$$

If $\theta / 2<\varphi<(\pi+\theta) / 2$, then $\sin \varphi \geqslant \sin \theta / 2$ when $\varphi \leqslant \pi / 2$ and $\sin \varphi$ $\geqslant \sin (\pi+\theta) / 2=\sin (\pi-\theta) / 2$ when $\varphi \geqslant \pi / 2$, and so

$$
\begin{aligned}
& |(r \cos t-1) \sin \theta \cos \varphi| \\
& \quad \leqslant(1-r \cos t) \sin \theta\left(\frac{1-\cos \Psi}{(1-r \cos t) \sin \theta \sin \varphi}\right)^{1 / 2} \\
& \quad \leqslant 2^{1 / 2}(1-\cos \Psi)^{1 / 2}\left(\frac{\sin \theta}{\sin \varphi}\right)^{1 / 2} \leqslant C(1-\cos \Psi)^{1 / 2} .
\end{aligned}
$$

Thus inequality (5.6) holds.

From (4.2), (5.4), (5.6) it follows that if $\alpha>\beta>-\frac{1}{2}$ and $\Phi_{0}<\Phi$ then

$$
\begin{aligned}
\int \mid \sigma_{n}^{\gamma}(\theta, \Phi) & -\sigma_{n}^{\gamma}\left(\theta, \Phi_{0}\right) \mid d \mu(\theta) \\
= & \int\left|\int_{\Phi_{0}}^{\Phi} \frac{\partial}{\partial \varphi} \sigma_{n}^{\gamma}(\theta, \varphi) d \varphi\right| d \mu(\theta) \\
= & \int\left|\int_{\Phi_{0}}^{\Phi} \int_{0}^{\pi} \int_{0}^{1} \frac{\partial \sigma_{n}^{\gamma}(\Psi)}{\partial \varphi} d m(r, t) d \varphi\right| d \mu(\theta) \\
= & \int\left|\int_{\Phi_{0}}^{\Phi} \int_{0}^{\pi} \int_{0}^{1} \frac{d \sigma_{n}^{\gamma}(\Psi)}{d \cos \Psi} \frac{\partial \cos \Psi}{\partial \varphi} d m(r, t) d \varphi\right| d \mu(\theta) \\
& \leqslant C \iint_{\Phi_{0}}^{\Phi} \int_{0}^{\pi} \int_{0}^{1}(1-\cos \Psi)^{1 / 2}\left|\frac{d \sigma_{n}^{\gamma}(\Psi)}{d \cos \Psi}\right| d m(r, t) d \varphi d \mu(\theta) \\
= & C \iint_{\Phi_{0}}^{\Phi} \int(1-\cos \psi)^{1 / 2}\left|\frac{d \sigma_{n}^{\gamma}(\psi)}{d \cos \psi}\right| K(\theta, \varphi, \psi) d \mu(\psi) d \varphi d \mu(\theta) \\
= & C \iint_{\Phi_{0}}^{\Phi}(1-\cos \psi)^{1 / 2}\left|\frac{d \sigma_{n}^{\gamma}(\psi)}{d \cos \psi}\right|\left\{\int K(\theta, \varphi, \psi) d \mu(\theta)\right\} d \varphi d \mu(\psi),
\end{aligned}
$$

which by means of (4.3) gives

$$
\begin{aligned}
\int \mid \sigma_{n}^{\gamma}(\theta, \varphi)- & \sigma_{n}^{\gamma}\left(\theta, \varphi_{0}\right) \mid d \mu \\
& \leqslant C\left|\varphi-\varphi_{0}\right| \int(1-\cos \psi)^{1 / 2}\left|\frac{d \sigma_{n}^{\gamma}(\psi)}{d \cos \psi}\right| d \mu(\psi)
\end{aligned}
$$

for $0 \leqslant \varphi, \varphi_{0} \leqslant \pi$. 
Similarly, the case $\alpha=\beta=-\frac{1}{2}$ of (5.9) follows by using (5.3), and the cases $\alpha=\beta>-\frac{1}{2}$ and $\beta=-\frac{1}{2}<\alpha$ follow by using the fact [13, p. 131] that (5.4) also holds in these two cases with the measure $\operatorname{dm}(r, t)$ replaced by the measures

$$
\frac{\Gamma(\alpha+1)}{\Gamma\left(\frac{1}{2}\right) \Gamma\left(\alpha+\frac{1}{2}\right)} \delta(1-r)(\sin t)^{2 \alpha} d r d t
$$

and

$$
\frac{\Gamma(\alpha+1)}{\Gamma\left(\frac{1}{2}\right) \Gamma\left(\alpha+\frac{1}{2}\right)}\left(1-r^{2}\right)^{\alpha-1 / 2}(\delta(t)+\delta(\pi-t)) d r d t,
$$

respectively, where $\delta(t)$ represents Dirac's delta function. Note, in particular, that when $\alpha=\beta>-\frac{1}{2}$ this is equivalent to using the Gegenbauer product formula (see $[8,3.15(20)])$

$$
\begin{aligned}
& R_{n}^{(\alpha, \alpha)}(\cos \theta) R_{n}^{(\alpha, \alpha)}(\cos \varphi) \\
& \quad=\frac{\Gamma(\alpha+1)}{\Gamma\left(\frac{1}{2}\right) \Gamma\left(\alpha+\frac{1}{2}\right)} \int_{0}^{\pi} R_{n}^{(\alpha, \alpha)}(\cos \theta \cos \varphi+\sin \theta \sin \varphi \cos t)(\sin t)^{2 \alpha} d t
\end{aligned}
$$

in place of the Koornwinder product formula.

By using [16, (4.21.7)]

$$
(d / d x) P_{n}^{(\alpha, \beta)}(x)=\frac{1}{2}(n+\alpha+\beta+1) P_{n-1}^{(\alpha+1, \beta+1)}(x)
$$

and the method which Bonami and Clerc [3] used to derive the estimates (5.2) it is easily shown that if $\gamma \leqslant \alpha+\frac{3}{2}$ (and in fact for a larger range) then

$$
\left|\frac{d \sigma_{n}^{\gamma}(\psi)}{d \cos \psi}\right| \leqslant \begin{cases}C n^{2 \alpha+4}, & 0 \leqslant \psi \leqslant \pi / 2, \\ C n^{\alpha+3 / 2-\gamma} \psi^{-\alpha-\gamma-5 / 2}, & 2 / n \leqslant \psi \leqslant \pi / 2, \\ C n^{\alpha+3 / 2-\gamma}(\pi-\psi)^{-\beta-3 / 2}, & \pi / 2 \leqslant \psi \leqslant \pi-2 / n, \\ C n^{\alpha+\beta+3-\gamma}, & \pi / 2 \leqslant \psi \leqslant \pi,\end{cases}
$$

when $\alpha \geqslant \beta \geqslant-\frac{1}{2}$. These estimates give

$$
\int(1-\cos \psi)^{1 / 2}\left|\frac{d \sigma_{n}^{\gamma}(\psi)}{d \cos \psi}\right| d \mu(\psi) \leqslant C n,
$$

which, with (5.9), completes the proof of Proposition 1. Inequality (5.10) and the last inequality in (5.1) can also be proved by repeating the steps in Szegö's proof $[16, \S 9.41]$ of the boundedness of the sequence of Lebesgue constants for Jacobi series. 
6. The Poisson kernel. If $\sum_{0}^{\infty} a_{n}$ is any absolutely convergent series and

$$
k(\theta, \varphi)=\sum_{n=0}^{\infty} a_{n} R_{n}(\cos \theta) R_{n}(\cos \varphi),
$$

then by the methods in (5.1) and (5.8) it follows for $\alpha \geqslant \beta \geqslant-\frac{1}{2}$ that

$$
\int|k(\theta, \varphi)| \cdot|\theta-\varphi|^{\varepsilon} d \mu(\theta) \leqslant \int|k(\psi)| \psi^{\varepsilon} d \mu(\psi), \quad \varepsilon \geqslant 0,
$$

and

(6.2) $\int\left|k(\theta, \varphi)-k\left(\theta, \varphi_{0}\right)\right| d \mu(\theta) \leqslant C\left|\varphi-\varphi_{0}\right| \int(1-\cos \psi)^{1 / 2}\left|\frac{d k(\psi)}{d \cos \psi}\right| d \mu(\psi)$, where $k(\psi)=k(\psi, 0)$. In particular, for the Poisson kernel $W_{r}(\theta, \varphi), 0<r$ $\leqslant 1$, we have

$$
\int W_{r}(\theta, \varphi)|\theta-\varphi|^{\varepsilon} d \mu(\theta) \leqslant \int W_{r}(\psi) \psi^{\varepsilon} d \mu(\psi), \quad \varepsilon \geqslant 0,
$$

and

$$
\begin{aligned}
\int \mid W_{r}(\theta, \varphi) & -W_{r}\left(\theta, \varphi_{0}\right) \mid d \mu(\theta) \\
& \leqslant C\left|\varphi-\varphi_{0}\right| \int(1-\cos \psi)^{1 / 2}\left|\frac{d W_{r}(\psi)}{d \cos \psi}\right| d \mu(\psi),
\end{aligned}
$$

where $W_{r}(\psi)=W_{r}(\psi, 0)$. Thus to prove (1.6) and the inequality (see [7, p. 249])

$$
\int W_{r}(\theta, \varphi)\left(\frac{|\theta-\varphi|}{r}\right)^{\varepsilon} d \mu(\theta) \leqslant C_{\varepsilon}, \quad 0 \leqslant \varepsilon<1,
$$

we need but show that

$$
\int(1-\cos \psi)^{1 / 2}\left|\frac{d W_{r}(\psi)}{d \cos \psi}\right| d \mu(\psi) \leqslant \frac{C}{r}
$$

and

$$
\int W_{r}(\psi)\left(\frac{\psi}{r}\right)^{\varepsilon} d \mu(\psi) \leqslant C_{\varepsilon}, \quad 0 \leqslant \varepsilon<1 .
$$

Note that in (6.3) and (6.7), unlike in the Cesàro kernel case (5.1), it was not necessary to use the absolute value of the Poisson kernel since this kernel is nonnegative (it has recently been proved [11] that $\sigma_{n}^{\gamma}(\theta, \varphi) \geqslant 0, n=0,1, \ldots$, for $\alpha \geqslant \beta \geqslant-\frac{1}{2}$ if and only if $\gamma \geqslant \alpha+\beta+2$ ). 
The estimates needed to prove (6.6) and (6.7) are much easier to derive than the corresponding ones for the Cesàro kernel case since we can use the hypergeometric representation

$$
\begin{aligned}
W_{r}(\psi)= & \frac{\Gamma(\alpha+\beta+2)}{\Gamma(\alpha+1) \Gamma(\beta+1)} \cdot \frac{r}{(2-r)^{\alpha+\beta+2}} \\
& \times F\left[\frac{\alpha+\beta+2}{2}, \frac{\alpha+\beta+3}{2} ; \beta+1 ; \frac{2(1-r)(1+\cos \psi)}{(2-r)^{2}}\right],
\end{aligned}
$$

which is a consequence of Bailey's formula [1, (2.3)]. From (6.8) we have

$$
\begin{aligned}
\frac{\partial W_{r}(\psi)}{\partial \cos \psi}=\frac{C r(1-r)}{(2-r)^{\alpha+\beta+2}} F\left[\frac{\alpha+\beta+4}{2}\right. & ; \frac{\alpha+\beta+5}{2} ; \\
\beta+2 & \left.; \frac{2(1-r)(1+\cos \psi)}{(2-r)^{2}}\right],
\end{aligned}
$$

where $C$ depends only on $\alpha$ and $\beta$. Thus

$$
\begin{aligned}
W_{r}(\psi)= & \frac{C r(2-r)^{\alpha-\beta+1}}{\left\{r^{2}+2(1-r)(1-\cos \psi)\right\}^{\alpha+3 / 2}} \\
& \times F\left[\frac{\beta-\alpha}{2}, \frac{\beta-\alpha-1}{2} ; \beta+1 ; \frac{2(1-r)(1+\cos \psi)}{(2-r)^{2}}\right]
\end{aligned}
$$

and

$$
\begin{aligned}
\frac{\partial W_{r}(\psi)}{\partial \cos \psi}= & \frac{C r(1-r)(2-r)^{\alpha-\beta+1}}{\left\{r^{2}+2(1-r)(1-\cos \psi)\right\}^{\alpha+5 / 2}} \\
& \times F\left[\frac{\beta-\alpha}{2}, \frac{\beta-\alpha-1}{2} ; \beta+2 ; \frac{2(1-r)(1+\cos \psi)}{(2-r)^{2}}\right]
\end{aligned}
$$

by means of the transformation formula $[8,2.9(2)]$

$$
F[a, b ; c ; z]=(1-z)^{c-a-b} F[c-a, c-b ; c ; z] .
$$

Since the series $F[a, b ; c ; z]$ converges [8, \$2.1.1] for $z=1$ and hence uniformly for $0 \leqslant z \leqslant 1$ when $c-a-b>0, c>-1$, it follows that the hypergeometric series on the right sides of (6.9) and (6.10) are uniformly bounded; so that

$$
W_{r}(\psi) \leqslant \frac{C r}{\left(r^{2}+(1-r) \psi^{2}\right)^{\alpha+3 / 2}}
$$

and 


$$
\left|\frac{\partial W_{r}(\psi)}{\partial \cos \psi}\right| \leqslant \frac{C r(1-r)}{\left(r^{2}+(1-r) \psi^{2}\right)^{\alpha+5 / 2}}
$$

for $\alpha, \beta>-1,0<r \leqslant 1,0 \leqslant \psi \leqslant \pi$. By integrating over the intervals $(0, r)$ and $(r, \pi)$ these estimates give (6.6) and (6.7), and hence (1.6) and (6.5) for $\alpha \geqslant \beta \geqslant-\frac{1}{2}$. Inequality (6.5) can be shown to also hold for $\alpha+\beta \geqslant-1,-1$ $<\beta<-\frac{1}{2}$, by using the integral representation for the product of Jacobi polynomials in [10].

Extensions of Theorem 1 and additional applications of the methods of this paper will be given elsewhere.

\section{REFERENCES}

1. W. N. Bailey, The generating function of Jacobi polynomials, J. London Math. Soc. 13 (1938), 8-12.

2. H. Bavinck and W. Trebels, On $M_{p}^{q}$ multipliers for Jacobi expansions (to appear).

3. A. Bonami and J.-L. Clerc, Sommes de Cesàro et multiplicateurs des développements en harmoniques sphériques, Trans. Amer. Math. Soc. 183 (1973), 223-263. MR 49 \#3461.

4. L. S. Bosanquet, Note on the Bohr-Hardy theorem, J. London Math. Soc. 17 (1942), 166-173. MR 4, 194.

5. R. R. Coifman and G. Weiss, Analyse harmonique non-commutative sur certains espaces homogenes, Lecture Notes in Math., vol. 242, Springer-Verlag, Berlin and New York, 1971.

6. W. C. Connett and A. L. Schwartz, A multiplier theorem for ultraspherical series, Studia Math. 51 (1974), 51-70. MR 50 \# 10674.

7. - A multiplier theorem for Jacobi expansions, Studia Math. 52 (1975), 243-261.

8. A. Erdélyi, W. Magnus, F. Oberhettinger and F. G. Tricomi, Higher transcendental functions, vol. I, McGraw-Hill, New York, 1953. MR 15, 419.

9. G. Gasper, Positivity and the convolution structure for Jacobi series, Ann. of Math. (2) 93 (1971), 112-118. MR 44 \# 1852.

10. - Banach algebras for Jacobi series and positivity of a kernel, Ann. of Math. (2) 95 (1972), 261-280. MR 46 \#9634.

11. - Positive sums of the classical orthogonal polynomials, SIAM J. Math. Anal. (to appear).

12. L. Hörmander, Estimates for translation invariant operators in $L^{p}$ spaces, Acta Math. 104 (1960), 93-140. MR 22 \# 12389.

13. T. Koornwinder, Jacobi polynomials. II, An analytic proof of the product formula, SIAM J. Math. Anal. 5 (1974), 125-137.

14. J. Marcinkiewicz, Sur les multiplicateurs des séries de Fourier, Studia Math. 8 (1939), 78-91.

15. B. Muckenhoupt and E. M. Stein, Classical expansions and their relation to conjugate harmonic functions, Trans. Amer. Math. Soc. 118 (1965), 17-92. MR 33 \#7779.

16. G. Szegö, Orthogonal polynomials, 3rd ed., Amer. Math. Soc. Colloq. Publ., vol. 23, Amer. Math. Soc., Providence, R.I., 1967. (1st ed., 1939; MR 1, 14)

17. W. Trebels, Multipliers for $(C, \alpha)$-bounded Fourier expansions in Banach spaces and approximation theory, Lecture Notes in Math., vol. 329, Springer-Verlag, Berlin and New York, 1973.

18. A. Zygmund, Trigonometric series, vol. 2, 2nd rev. ed., Cambridge Univ. Press, New York, 1959. MR 21 \#6498.

Department of Mathematics, Northwestern University, Evanston, Illinois 60201

FAChbereich Mathematik, TH Darmstadt, D-6100 Darmstadt, West Germany 